\title{
The Gamification in the Design of Computational Applications to Support the Autism Treatments: An Advance in the State of the Art
}

\author{
Gustavo Eduardo Constain M. ${ }^{1}$ (10, César Collazos O. ${ }^{2}$ (D), \\ and Fernando Moreira ${ }^{3,4(\triangle)}$ (D) \\ ${ }^{1}$ University of Cauca, National and Distance Open University, \\ Popayán, Colombia \\ gconsta@unicauca.edu.co \\ ${ }^{2}$ University of Cauca, Popayán, Colombia \\ ccollazo@unicauca.edu.co \\ 3 REMIT, IJP, Universidade Portucalense,
}

Rua Dr. António Bernardino Almeida, 541-619, 4200-072 Porto, Portugal fmoreira@upt.pt

${ }^{4}$ IEETA, Universidade de Aveiro, Aveiro, Portugal

\begin{abstract}
This paper presents the results found when consulting literature, with the purpose of elaborating the state of the art related to the use of gamification techniques in the development of computational applications that support the treatment of the Autism Spectrum Disorder (ASD). The research focuses on the results of using some models for the design of inclusive software, the steps to elaborate them and the possibility of adapting game techniques; so that its use can be modeled and adapted in a future framework for the design of specific applications for the autism management. Due to its characteristic, the game involves an intrinsic motivation, and a number of sensations that facilitate the development of emotions in the people who interact, that is why the study carried out seeks to find the importance of linking these techniques to the interior of the treatments of ASD, especially when it is intended to develop skills, such as self-recognition and social performance in children suffering from autism. With this article, we also want to propose an initial design framework for inclusive computational applications, based on gamification, related to the achievement of emotional skills in children with ASD.
\end{abstract}

Keywords: EmoTEA - Gamification $\cdot$ MDA Framework $\cdot$ 6D model $\cdot$ Autism Spectrum Disorder $\cdot$ MPIu+a model

\section{Introduction}

This paper is part of the development of a research project that seeks the creation of a framework, with some recommendations, for the design of inclusive computational applications applied to the treatment of Autism Spectrum Disorder (ASD). For the achievement of this purpose, it is important to identify the elements that are basic for

(C) Springer Nature Switzerland AG 2019

Á. Rocha et al. (Eds.): WorldCIST'19 2019, AISC 932, pp. 195-205, 2019.

https://doi.org/10.1007/978-3-030-16187-3_19 
the inclusive computer design, but also, that what is implemented is part of the factors used in the treatment of autism, for example, the improvement of visual communication and social performance [1]. This is the case of 'play' as a basic element for the development of emotional skills in autistic children, as long as it is part of a professional treatment, applied among therapists, educators and the child's family.

The use of play strategies within the activities of the treatment of autism is quite usual; however, current therapeutic trends seek to improve the impact on the development of emotional and social skills with elements of serious games, implemented to through computer applications [1]. Therefore, by including some elements for treatment in computerized scenarios, called "gamification" or "serious games", has given positive results. However, it generates new concerns for therapists and the computer community. This is due to specific needs that vary from case to case of ASD, although they may appear similar.

In this paper, an approach to the state of the art presented that contributes to the construction of computational tools used as support in the treatment of autism, from a gamification based approach. It starts from the study of two models of computer application design for gaming and that related to the development of applications to support treatments of some type of disability. Among these are the MDA Framework and the 6D Method, which contain different frameworks for game design. While MDA provides the Mechanical, Dynamic and Aesthetic design of the application, 6D defines the phases by which we can build an inclusive application. Likewise, the MPIu+a model studied as a generic mechanism for the design of inclusive computing applications. Finally, we seek to propose an integrating model of design strategies for inclusive gamified applications and their use in inclusive environments that support the treatment of ASD, which corresponds to the EmoTEA Framework made by the authors of the document.

\section{Related Work}

The research work that originates this article seeks, on the one hand, the detailed knowledge of the characteristics of the ASD as well as the treatment options used; and on the other hand, the alternatives for the design of inclusive computational applications, investigating those that directly focus on cases of autism. Recently, a case study has shown us the direct relationship between the use of computer applications and the development of emotions in five children between 5 and 12 years of age (Fig. 1).

In this case, the study consisted of the evaluation of the impact of the use of computer tools in the generation of some emotional reaction in children diagnosed with autism. This study carried out with children from two educational institutions, with whom an exploratory study applied to find the relationship between autism and the use of learning applications for these cases.

Now, a next stage of the project requires an in-depth study of the types of inclusive computer applications most used in the treatment of autism and the determination of its functionality according to the most commonly used therapeutic requirements in the case study. This would allow identifying the characteristics of interaction, emotional 


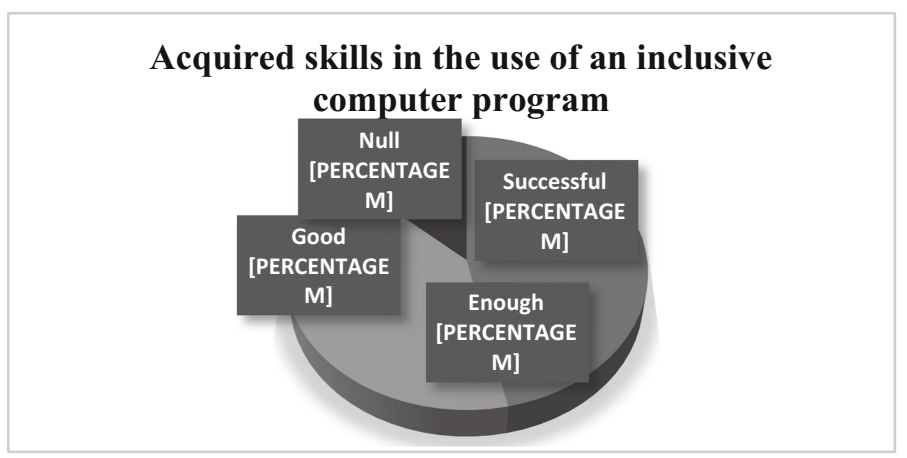

Fig. 1. Use of computer applications and emotional development

and social development, as well as communication between Therapist-Autistic ChildFamily, this as part of an integrated model that improves the current treatment results.

\section{Methodology}

We opted for a systematic review (Fig. 2) to collect information of interest on technical issues considered important. The importance of the mapping of the systematic review is found in the structure and in the steps, it proposes to carry out the searches in an organized and methodological way, which helps to generate reliable results in the research [2].

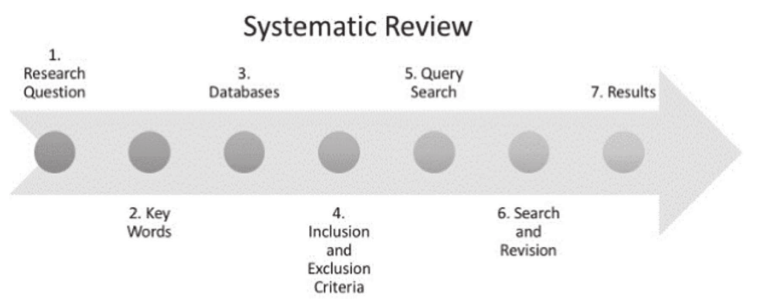

Fig. 2. Process for systematic review

In the process of systematic review [5], we follow the following steps: 1-Statement of research questions. 2-Definition of keywords for searches in English and Spanish. 3-Definition of databases to consult. 4-Definition of criteria for inclusion and exclusion of articles, and the search time interval. 5-Definition of advanced search chains in each of the databases. 6-Realization of search process and review of articles. 7-General review and Writing of found results. In this case, the research was specifically oriented to the design models of computationally inclusive applications and those that use gamification strategies and that could use as support for the treatment of the autistic spectrum syndrome. 


\section{Research Questions}

RQ1: What types of treatment, with computational support, recently applied to the emotional management of autistic spectrum disorder - ASD?

RQ2: What possible gamification strategies used in computer applications to support the emotional treatment of autism?

RQ3: Is there any integration model between the clinical treatment of autistic spectrum disorder - ASD and the use of gamified applications?

Keywords: For the systematic review, the search for the key words in English and Spanish defined to include greater results of the searches and to allow a more complete revision in the databases. The words queries are the following: Abilities, Autism, Treatment, Emotions, Model and Gamification.

Databases: To make the research we use the following databases: Google Scholar, SCOPUS, Web of Science, IEEE Xplore and Science Direct.

Search Strings: We defined a general search chain based on the general concepts of the search time and that allowed us to answer the research questions posed. (("gamification*" OR "serious game") OR ("computation*")) AND ("treatment") AND ("autism*" OR "ASD" OR "syndrome").

Search Process: Were found 496 articles in the databases and with them, the review process was started. In the first place, a general review carried out to find the articles, book chapters, etc., 53 articles repeated, originated by the different searches in the databases and by different search chains. Then, a revision of the titles and the summary of the works carried out. The general summary of the accepted works detailed in Fig. 3.

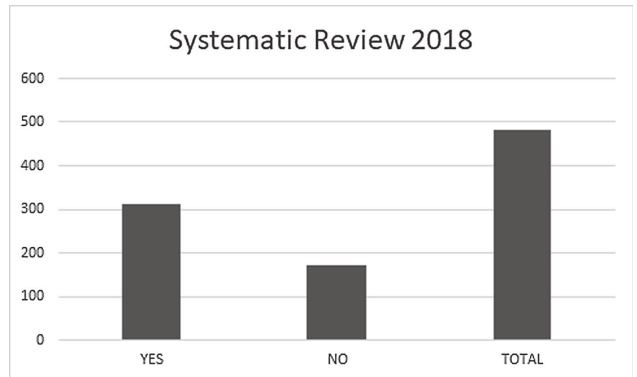

Fig. 3. Papers accepted in the systematic review

\section{State of the Art}

The review of literature allowed the identification of the most relevant studies for the purposes of the general project, which deepens in the knowledge of current treatments for autism, the existence of treatment activities based on the use of computer applications and the relationship between the use of gamification techniques and the development of emotional skills. In the same way, we study two models of design of gamified applications, viable of use for the particular purposes, and a model of design of inclusive applications. 


\subsection{Applied Studies}

\section{Related to the Treatment of ASD}

It is clear that technology is a great ally to help in the development of people with disorders such as autism. In this context, there is a proliferation of initiatives aimed at the therapeutic support of this population, but adjusted to particular cases that have generated the formulation of projects in many cases, and collections of mobile applications aimed at communities that work in the treatment of this disorder.

The treatment alternatives experienced within the therapeutic circle has varied to look for complementary alternatives valid to the medicated management of children suffering from ASD [4]. Thus, for example, it been proven with the use of computer applications to facilitate the child's development of skills for concentration and learning activities in their daily lives. This therapy has used for its results in the medium or long term and it has varied in its application from the use of printed pictograms, digitized tablets, or computers to innovative therapies using trained dogs for children to copy their behavior in front of certain situations for which they want to appropriate their behavior, or simply because they calm their emotional sensations [5].

According to the above, we have explored the applications that most mentioned in the literature for work with autism, and that are known as Alternative and Augmentative Communication Systems (SAACs) [6].

In the initial exploration of the websites of the authors, it was possible to verify that, although there is a direct relationship in the conceptual management and presentation of the pictograms within the possibilities of use in cases of ASD, it is not directly visible its use as a complement to a therapeutic treatment accompanied by clinical experts. Consequently, we would have to resort to direct contact with the authors to request timely information about the technical details of implementation of these solutions and look for some type of case related to aspects of treatment design making use of each particular application.

\section{Relationship Between Gamification and Development of Emotional Skills}

The literature exploration, throws interesting deductions regarding the formulation of new therapeutic initiatives and that has originated programs such as the use of mobile devices with NFC technology [7]. Notwithstanding the above, no research have been found related to the combined application of the above elements, that is, the use of software tools that use NFC technology for the presentation of animated pictograms, in addition to the inclusion of elements of serious games that support the current treatment of children with ASD, or even, with other functional diversity, so it would be a contribution to new practices of inclusive education in this area of knowledge.

With respect to the model for the design of computational applications, that support the treatments of ASD, although in the selected literature the use of applications within some treatments is found with important results for the improvement of the behavior of children with ASD [4, 8], it is evident that it is not possible to generalize a treatment for various cases of autism that have been diagnosed, therefore, a specific treatment should be designed for each case and in this sense it would also have to make specific adjustments to the computational application that is designed from the focus of the game, interaction and accessibility principles [9]. 
Consequently, we seek to explore the advantages that the development of emotional skills involves the use of game techniques within therapeutic alternatives, this is the use of gamification within the design strategy of computational applications, where the use of these techniques for the innovation of therapeutic proposals facilitates the intrinsic motivation of the child with ASD (who learns) and increases the expected results [10, 12]. For the gamified design, the ARCS basic design model (Fig. 4) would be used to determine the levels of Satisfaction, Safety, Attention and Relevance [13] that are determined in a collaborative manner between the actors involved in the design: Therapist, Family members, Engineers and of course the factors identified in the child with ASD.

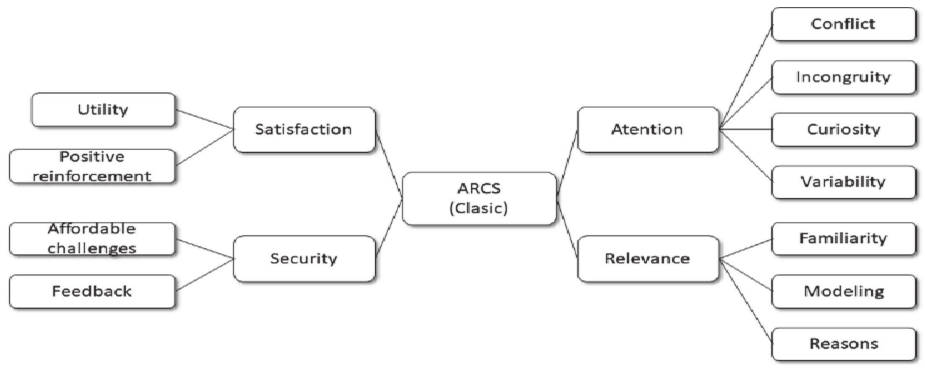

Fig. 4. Classic gamified design model

\section{Related to the Design of Applications with the MDA Model}

Because of the relationship between gamification and game, it is possible to use the same tools used for the development and creation of this type of applications. One such tool is the MDA Framework, one of the most common frameworks for game design [11]. This framework is a formal approach to understanding games, and its function is to be a design tool and a study tool. MDA allows approaching the design of the games in a methodical and iterative way. In addition, this approach allows conceptualizing the dynamic behavior of the games, thus facilitating the creation of iterative development techniques, allowing the early identification of undesirable results and rethinking development strategies according to what was expected (Fig. 5).

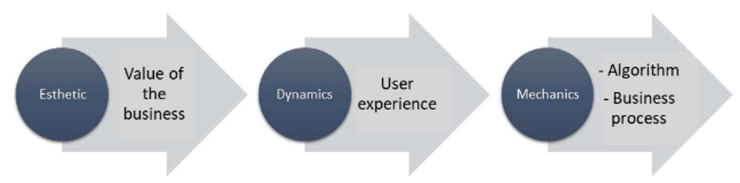

Fig. 5. MDA Framework

\section{Related to the Design of Applications with the Model $6 D$}

It is another working framework consisting of six (6) phases designed to design game type applications: (1) Definition of system objectives; (2) Definition of desired 
behaviors; (3) Description of the players; (4) Design of activity cycles; (5) Fun elements added; (6) Implementation of appropriate tools.

In addition to this type of architectural aspects, when gamified development is applied in contexts of support for therapies of syndromes such as ASD, it should be based on the collaborative gamification approach that should allow the mediation or accompaniment of an expert (therapist or specialized doctor) and guarantee the interactivity between all the participants of the experience: relatives and people supporting the computational development. All of them have the advantage of supported under HTML5 languages, CSS3, PHP and SQL support, which makes them compatible with many current internet devices and browsers.

\section{Related to the Design of Applications with the MPIu+a Model}

The Usability and Accessibility Engineering Process Model-MPIu+a, seeks to cover the aspects related to the Design of Interactive Systems Centered on the User (UCD) contemplating all its phases of realization (Fig. 6): Analysis, Design, Implementation, Launching, Prototyping and Evaluation [14, 15].

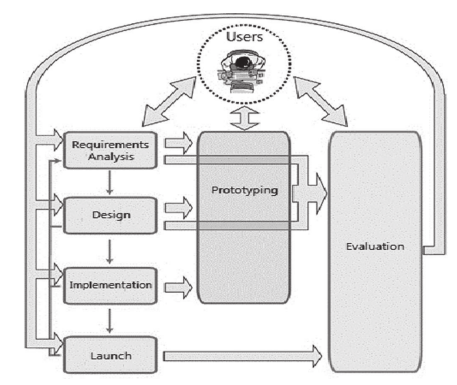

Fig. 6. MPIu+a model

Initially, the development of the model of inclusive computer applications from the concepts related to the disability linked to the ASD is framed and specifically in this case, it should focus on the development of intrapersonal skills (such as motivation) and interpersonal skills (for example, social skills) related to emotional intelligence that is what is sought in the research. This has to do with the conceptual organization of the project within the aspects of Software Engineering with the basic principles of Usability Engineering and Accessibility, providing a methodology that is able to guide development teams during the process of implementing a certain gamified, interactive and inclusive system.

The requirements of an interactive system usually refer to the functional component of the software leaving other aspects beyond the scope of the system, such as who will be the users and the use they make of the system. Due to the above, this phase of the process model is based on the Engineering Requirements and the quality model defined in the ISO/IEC 25010 standard, which describes the quality of the system requirements in the initial stages of the cycle life, referring mainly to the external view and view of the user (functionality, performance, security, maintainability, compatibility, portability 
and especially usability) rather than in reference to the internal or functional quality of the implementation, which is to which only the developers refer.

\subsection{New Design Framework for Inclusive Applications for TEA (EmoTEA)}

The engineering models of the requirements for the case of inclusive applications for the treatment of autism, add new factors to be taken into account, which should guarantee the development of applications with a much better degree from the functional point of view, that is, from its usability and its accessibility for children with ASD.

During the development of a computational application, once the functionalities that the system should cover along with the rest of its characteristics derived from the context of the interaction are resolved, the design of the activity and the design of the information are continued. These make up the general process of designing this interaction. In addition to this, the quality attributes mentioned by ISO 25010 and the playability characteristics required for gamified applications must be applied; therefore, within its architectural definition, a cycle of evaluation (or improvement) of the application to be developed must be included (Fig. 7). This is where the MPIu+a models and the already mentioned MDA and/or 6D design frameworks would be integrated.

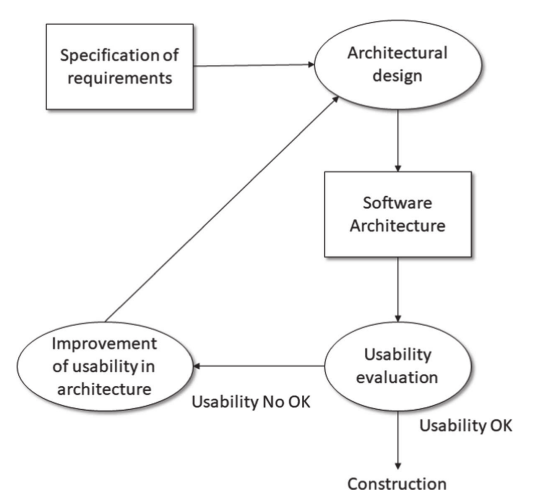

Fig. 7. Design of applications for usability

The above guarantees that both functional and non-functional requirements are met from the beginning of the design of the applications and not until the end that the tests are performed with children who immersed in an ASD treatment.

Once defined the functional requirements, a model of integration of the necessary elements is proposed to allow the design of computational applications with characteristics of development of emotional skills (Learning and treatment management system), tools for the co-creation of computer-assisted therapies, the product of gamified software and alternatives for interaction and communication between the therapist and the family of the child with ASD (Fig. 8). 


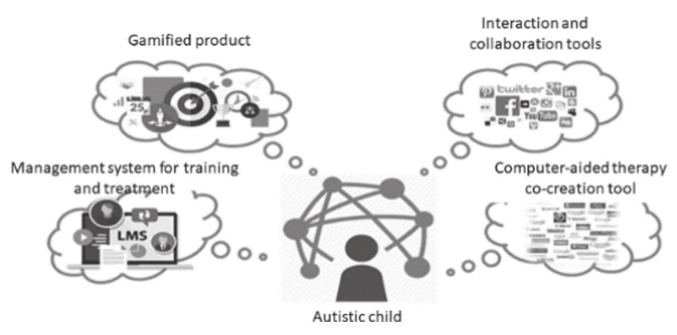

Fig. 8. Conceptual diagram of the EmoTEA Framework

\section{Results}

From a clinical approach, we learned that there are no two equal cases of ASD, therefore the definition of a treatment with computational elements must be specific for each child in particular. In this same sense, the development of emotional skills is a key factor in the treatment of autism, especially self-recognition and empathy, to facilitate the social interaction of the child. In this sense, there are diverse experiences of using specialized software tools for autism, which yield important results of usability and design, that provide key elements for a framework of design of specific computational applications in autism treatments, in the which requires the permanent monitoring of the therapist and the construction at home of the activities to be carried out for different periods of time.

For the above, there are computational applications design models, such as MDA, 6D and MPIu+a, that provide help for autism, but these have generally been used autonomously and without rigorous follow-up by the clinical specialist or without linkage of the child's family in the follow-up of the treatment.

These same models facilitate the development of computer applications for contexts of application of gamified elements, but taking into account the final user for whom they must designed, i.e. children with autistic disorder. Regarding the design of these specialized software applications, there are models, patterns and generalized recommendations for the design of inclusive software, but these lack some particular elements for the treatment of syndromes such as ASD. The state of art and technology, finds the existence of design models of inclusive applications (such as MPIu+a) from which you can start the proposal to create a specific framework, based on humancomputer interaction, for the adaptation of inclusive computational applications, suitable to support the treatment of autism. This model of integration of inclusive computer applications and elements of autism treatment must be developed systematically and with a consequent validation of results from the most relevant usability and accessibility heuristics. 


\section{Discussion and Conclusions}

Currently in Colombia is more evident the number of people with some type of disability, especially ASD, which requires a psycho-educational treatment tailored to their condition and especially configurable according to the progress of their therapy of cognitive stimulation. In this same context, we have identified treatments that make use of computer applications as complementary support, but not exclusive, within the therapeutic alternatives. These are mainly based on the training of emotional and social skills through the automated presentation of images (pictograms) or the mental exercise search of the child with ASD. With the above scenario, we solve the first question proposed for this review of the technique related to the treatment of autism.

However, it is found that the design of computer applications to support the disabled in Colombia is still incipient and it would be to verify if this is perhaps because the software community requires more conceptual tools that allow the design of inclusive applications orderly and quality for this population. The systematic review of computational applications for the ASD treatment shows an initial number of qualitatively evaluable software programs, however, it is not easy to identify validity as a support to established clinical treatments. Thus, a framework for the design of inclusive computational applications for the treatment of ASD can start from the use of already established architecture patterns and the use of models of application of game techniques, such as MDA or 6D; however, these must be adjusted to the extent that the therapeutic and technical conditions require it.

According to the above, it is possible to formulate a framework that seeks the parameterization of the design of computer applications that support the treatment of children with ASD, especially for the development of some specific predefined skills, which contributes to the achievement of our second question for this activity. Finally, in order to answer our last question, we can say that the MPIu+a model can be taken as a guide model for the usability and accessibility engineering process par excellence, however in particular cases of disability such as autism spectrum disorder could require some methodological adjustment to achieve the desired objectives, especially if specific requirements such as the linking of game techniques (gamification) are needed or if the computational application is an active part of the clinical treatment suggested by medical experts. The results of this study should verified and evaluated through their development and application in some case studies selected for experimentation within the next phase of the project that gives rise to this article.

\section{References}

1. Constain, G., Collazos, C., Fardoun, H.: Use of HCI for the development of emotional skills in the treatment of Autism Spectrum Disorder: a systematic review. Springer (2018)

2. Mattsson, M., Petersen, K., Feldt, R., Mujtaba, S.: Systematic mapping studies in software engineering, December 2018. http://www.robertfeldt.net/publications/petersen_ease08_ sysmap_studies_in_se.pdf

3. Social Promotion Office: Situational Room for People With Disabilities. Ministry of Health and Social Protection, Republic of Colombia (2015) 
4. Villalta, R., Sánchez Cabaco, A., Villa Estevez, J.: Design of digital applications for people with ASD. Int. J. Dev. Educ. Psychol. 4(1), 291-297 (2013). National Association of Evolutionary and Educational Psychology of Children, Adolescents and Seniors, Badajoz, Spain

5. Sutton, H.: Therapy dogs can be successful motivating students with autism spectrum disorder. Disabil. Compliance High. Educ. 22(7) (2017). Wiley Periodicals, Inc., A Wiley Company

6. Echenguia Cudolá, J.: Augmentative and alternative communication systems for the treatment of children with Autism Spectrum Disorder. 14(28), (2016). Universidad Católica de Córdoba, Argentina

7. Constain, G., Moreira, F., Collazos, C.: Use of HCI for the development of emotional skills in the treatment of Autism Spectrum Disorder: a systematic review. In: 13 ${ }^{\text {a }}$ Iberian Conference on Information Systems and Technologies (2018)

8. Lozano, J., Ballesta, J., Alcaraz, S., Cerezo, M.: Information and communication technologies (ICT) in the teaching and learning process of students with autistic spectrum disorder (ASD). Fuentes Mag. 14, 193-208 (2016). University of Murcia, Spain

9. Vélez, M.: TEACCH program: proposal of psychoeducational intervention in people with ASD. Cadiz University (2017)

10. Constain, M.G., Mora, P.A., Santiago, M.J.: Use of gamification for the development of emotional intelligence skills: a theoretical and experiential approach. In: IV Ibero-American Conference on Human-Computer Interaction (2018)

11. Zicherman, G., Cunningham, C.: Gamification by Design. O'reilly Media Inc., Toronto

12. Pelegrina del Río, M., Tejeiro, R.: The psychology of video games: a research model. Aljibe. Accessed 10 Jan 2019

13. Hunicke, R., LeBlanc, M., Zubek, R.: MDA: a formal approach to game design and game research. In: Proceedings of the Challenges in Game AI Workshop, 19th National Conference on Artificial Intelligence. AAAI Press, San José. http://www.cs.northwestern. edu/ hunicke/MDA.pdf. Accessed 10 Jan 2019

14. Liu, W.: Natural user interface-next mainstream product user interface. In: 2010 IEEE 11th International Conference on Computer-Aided Industrial Design \& Conceptual Design (CAIDCD). Accessed 10 Jan 2019

15. Granollers, T.: MPIu+a a methodology that integrates software engineering, humancomputer interaction and accessibility in the context of multidisciplinary development teams. Accessed 10 Jan 2019 